\title{
Human T-Lymphotrophic Virus Type-I: A Unique Association with Myelopathy in Sjögren's Syndrome
}

\author{
Alexandria Voigt ${ }^{1}$ and Cuong $Q$ Nguyen ${ }^{1,2^{*}}$ \\ ${ }^{1}$ Department of Infectious Diseases and Pathology, College of Veterinary Medicine, University of Florida, 2015 SW 16 th Ave, Gainesville Florida, USA \\ ${ }^{2}$ Center for Orphan Autoimmune Disorders, University of Florida College of Dentistry, 1600 SW Archer Rd, Gainesville, Florida, USA
}

*Corresponding author: Cuong Q. Nguyen, PhD, Department of Infectious Diseases and Pathology, PO Box 110880, College of Veterinary Medicine, University of Florida, Gainesville, Florida 32611-0880 USA, Tel: 352-294-4180; Fax: 352-392-9704; E-mail: Nguyenc@ufl.edu

Rec date: Dec 15, 2014; Acc date: Dec 25, 2014; Pub date: Jan 05, 2015

Copyright: $\odot 2014$ Nguyen CQ, et al. This is an open-access article distributed under the terms of the Creative Commons Attribution License, which permits unrestricted use, distribution, and reproduction in any medium, provided the original author and source are credited.

\section{Editorial}

Microbes play the most intricate and crucial role in the education of the immune system. Exposure to pathogens elicits specific immune response to clear the infection. After elimination of infectious pathogens, the immune cells undergo apoptosis during the contraction phase, and the remaining cells return to the quiescent memory phase [1]. During the primary and secondary responses, some of the immune cells fail to distinguish self and non-self. The failure to discriminate self and non-self entities triggers autoimmunity [2]. For decades, mounting evidence has supported the associations between infectious agents and autoimmune diseases. Two notable examples are rheumatic fever with Streptococcus pyogenes infection [3] and multiple sclerosis with Epstein-Barr virus (EBV) and Influenza type A virus [4]. The renewed interest in linkage of infectious disease and autoimmunity has thrived with primary autoimmune diseases associated with secondary peripheral neuropathy such as Sjögren's syndrome $(\mathrm{SjS}) . \mathrm{SjS}$ is an autoimmune disease which is generally categorized by sicca symptoms, the presence of (anti-SSA or anti-SSB) autoantibodies, and/or lymphocytic infiltration into the salivary gland $[5,6]$. There are a number of viruses which have been shown to associate with primary SjS [7-10]. However, lymphotrophic virus-1 (HTLV-1), which is a retrovirus originally identified as an etiological agent in $\mathrm{T}$ cell leukemia [11], is hypothesized to be correlated with the manifestation of myelopathy in some SjS patients and whose treatment efficacy has been variable [12].

In cases of infection, onset of myelopathy has been acute with patients feeling neck pain followed by parathesia, numbness or pain in their extremities which in some cases is followed by paraplegia [13]. There is debate as to whether HTLV-1 infection can act as an environmental trigger to cause SjS, however it remains unclear. Patients may not actually know that they have SjS until they seek out medical attention for these symptoms and patients with SjS can develop myelopathy without HTLV-1 infection. Ironically, patients with SjS would not usually be tested for HTLV-1 unless myelopathy occurred and patients with HTLV-1 infection would not be tested for SjS unless myelopathy occurred; some patients with $\mathrm{SjS}$ test negative for anti-SSA or anti-SSB autoantibodies and may lead to a negative diagnosis when $\mathrm{SjS}$ is present.

In one study [11], of ten patients presenting with HTLV-1 associated myelopathy (HAM), six were diagnosed with SjS and two were suspected to have it. Since all of the HAM patients also showed lymphocytic infiltration in the salivary glands (whether anti-SSA or anti-SSB autoantibodies were present), it becomes difficult to diagnose $\mathrm{SjS}$ as that is one of the qualifying criteria. Either salivary gland infiltration is a symptom of HTLV-1 associated exclusively to HAM, or SjS patients exclusively exhibit HAM. Further examination and larger cohorts of patients would be necessary to confirm which of these two options is correct.

Use of animal models is another viable option for studying this relationship. In one transgenic rat model (HTLV-1 env-pX), the env$p X$ gene was inserted into the WKAH strain [14] and rats developed SjS-like symptoms along with an array of other symptoms characteristic of autoimmune diseases [15], but manifestation of myelopathy was not studied. Further examination of this line, focusing on myelopathy exclusively may yield an answer as to whether or not HAM is present only in SjS patients, but this may be difficult as they are prone to developing other autoimmune disorders. Complexing SjS with another autoimmune disorder may convolute the findings as they may either exacerbate or mask symptoms.

To further understand HAM moving forward, it will probably become necessary to correctly diagnose patients with or without SjS. For HAM in SjS patients combined treatment with a corticosteroid and anti-viral has shown improvement in the patients' condition without resolving the lesion [16]; reversal of parathesia, numbness, pain or paraplegia may occur. Further examination of HAM both in a clinical setting and utilizing animal models may be necessary to fully understand if and why HAM presents exclusively in SjS patients.

\section{References}

1. Green DR (2003) Overview: apoptotic signaling pathways in the immune system. Immunological reviews, 193: p. 5-9.

2. Robey E, Fowlkes BJ (1994) Selective events in T cell development. Annual review of immunology, 12: p. 675-705.

3. Dinkla K (2003) Rheumatic fever-associated Streptococcus pyogenes isolates aggregate collagen. The Journal of clinical investigation 111: p. 1905-1912.

4. Wucherpfennig KW, Strominger JL (1995) Molecular mimicry in T cellmediated autoimmunity: viral peptides activate human $\mathrm{T}$ cell clones specific for myelin basic protein. Cell, 80: p. 695-705.

5. Lee BH, Tudares MA, Nguyen CQ (2009) Sjogren's syndrome: an old tale with a new twist. Arch Immunol Ther Exp (Warsz) 57: p. 57-66.

6. Nguyen CQ, Peck AB (2009) Unraveling the pathophysiology of Sjogren syndrome-associated dry eye disease. Ocul Surf 7: p. 11-27.

7. Killedar SJ (2006) Early pathogenic events associated with Sjogren's syndrome (SjS)-like disease of the NOD mouse using microarray analysis. Laboratory investigation; a journal of technical methods and pathology 86: p. 1243-1260.

8. Nguyen CQ (2008) Identification of possible candidate genes regulating Sjogren's syndrome-associated autoimmunity: a potential role for TNFSF4 in autoimmune exocrinopathy. Arthritis Res Ther 10: p. R137.

9. Fleck M (1998) Murine cytomegalovirus induces a Sjogren's syndromelike disease in C57Bl/6-lpr/lpr mice. Arthritis and rheumatism 41: p. 2175-84. 
Citation: Voigt A, Nguyen CQ (2015) Human T-Lymphotrophic Virus Type-I: A Unique Association with Myelopathy in Sjögren's Syndrome. Clin Microbiol 4: e123. doi:10.4172/2327-5073.1000e123

Page 2 of 2

10. Fox RI (1992) Potential role of Epstein-Barr virus in Sjogren's syndrome and rheumatoid arthritis. J Rheumatol Suppl, 32: p. 18-24.

11. Nakamura H (1997) High prevalence of Sjogren's syndrome in patients with HTLV-I associated myelopathy. Ann Rheum Dis, 56: p. 167-72.

12. Hermisson M (2002) Myelopathy in primary Sjogren's syndrome: diagnostic and therapeutic aspects. Acta Neurol Scand 105: p. 450-3.

13. Williams CS, Butler E, Roman GC (2001)Treatment of myelopathy in Sjogren syndrome with a combination of prednisone and cyclophosphamide. Arch Neurol, 58: p. 815-9.
14. Yamazaki H (1997) A wide spectrum of collagen vascular and autoimmune diseases in transgenic rats carrying the env-pX gene of human T lymphocyte virus type I. Int Immunol 9: p. 339-46.

15. Yoshiki T (1997) Models of HTLV-I-induced diseases. Infectious transmission of HTLV-I in inbred rats and HTVL-I env-pX transgenic rats. Leukemia, 11 Suppl 3: p. 245-246.

16. Pot C (2006) Combined antiviral-immunosuppressive treatment in human T-lymphotrophic virus 1-Sjogren-associated myelopathy. Arch Neurol 63: p. 1318-1320. 